\title{
Transition Radiation as a Probe of the Chiral Anomaly
}

\author{
Xu-Guang Huang ${ }^{1,2}$ and Kirill Tuchin ${ }^{3}$ \\ ${ }^{1}$ Physics Department and Center for Particle Physics and Field Theory, Fudan University, Shanghai 200433, China \\ ${ }^{2}$ Key Laboratory of Nuclear Physics and Ion-beam Application (MOE), Fudan University, Shanghai 200433, China \\ ${ }^{3}$ Department of Physics and Astronomy, Iowa State University, Ames, Iowa 50011, USA
}

(Received 5 August 2018; published 2 November 2018)

\begin{abstract}
A fast charged particle crossing the boundary between chiral matter and vacuum radiates transition radiation. Its most remarkable features - the resonant behavior at a certain emission angle and the circular polarization of the spectrum - depend on the parameters of the chiral anomaly in a particular material or matter. Chiral transition radiation can be used to investigate the chiral anomaly in such diverse media as the quark-gluon plasma, Weyl semimetals, and axionic dark matter.
\end{abstract}

DOI: 10.1103/PhysRevLett.121.182301

Introduction.-Chiral matter - the matter containing chiral fermions-possesses a number of unique properties originating from the quantum phenomenon of the chiral anomaly. Those chiral materials that exist at room temperature, such as Weyl semimetals, can be studied with high precision. On the other hand, there are forms of chiral matter that exist only under extreme conditions, such as the quark-gluon plasma; their study requires novel approaches. In this Letter we argue that an informative insight into properties of chiral matter can be gained using the chiral analogue of transition radiation.

Transition radiation is emitted when a fast charged particle, i.e., a particle moving with energy much greater than the medium ionization energy, crosses the boundary between two media having different dielectric constants. This is a classical effect predicted by Ginzburg and Frank in 1945 [1] (reviewed in Ref. [2]) that has a number of practical applications. The quantum corrections were calculated in Refs. [3,4]. Transition radiation originates from the difference of the photon wave function on the two sides of the boundary. At high energies this is manifested in a variation of the plasma frequency, across the boundary.

In a chiral matter the photon dispersion relation is modified due to the chiral anomaly [5]. As a result, when a fast charged particle crosses the boundary between the chiral matter and vacuum it emits transition radiation, which we will refer to as chiral transition radiation. Its spectrum was recently derived by K. T. in Ref. [6] employing a method developed in Ref. [4]. It possesses distinctive features as compared to other forms of radiation by fast

Published by the American Physical Society under the terms of the Creative Commons Attribution 4.0 International license. Further distribution of this work must maintain attribution to the author(s) and the published article's title, journal citation, and DOI. Funded by SCOAP. particles in matter. Thus, chiral transition radiation can be employed to investigate the chiral anomaly in various forms of matter and materials as we explain in the forthcoming sections.

The spectrum.-The dispersion relation of a photon in a chiral medium can be most readily computed using the Maxwell-Chern-Simons theory [7-10], which is an effective low energy approximation of QED in a chiral medium. The gauge part of this theory reads

$$
\mathcal{L}=-\frac{1}{4} F_{\mu \nu}^{2}-\frac{c_{A}}{4} \theta \tilde{F}_{\mu \nu} F^{\mu \nu},
$$

where the pseudoscalar field $\theta$ encapsulates the effect of the chiral anomaly and $c_{A}$ is the anomaly coefficient. In practical applications one usually assumes that $\theta$ is either (i) spatially uniform and adiabatically time dependent $\dot{\theta} \neq 0$, or (ii) that it is time independent and slightly anisotropic $\boldsymbol{\nabla} \theta \neq 0$. The dispersion relation in each case takes the form [11-14]

$$
\omega^{2}=\boldsymbol{k}^{2}+\mu^{2}(\boldsymbol{k}, \lambda),
$$

where $\boldsymbol{k}$ is the photon momentum and $\lambda= \pm 1$ is its circular polarization. The parameter $\mu$ is a complex function of its arguments that is sensitive to the spatial or temporal variation of the $\theta$ field. In case (i) it reads

$$
\mu^{2}(\boldsymbol{k}, \lambda)=-\lambda \sigma_{\chi} k,
$$

where $\sigma_{\chi}=c_{A} \dot{\theta}$ is the chiral conductivity $[15,16]$. In case (ii) it takes the form

$$
\mu^{2}(\boldsymbol{k}, \lambda)=\frac{1}{2} b^{2}-\lambda \operatorname{sgn}(\boldsymbol{k} \cdot \boldsymbol{b}) \sqrt{(\boldsymbol{k} \cdot \boldsymbol{b})^{2}+\frac{1}{4} b^{4}},
$$

where $\boldsymbol{b}=c_{A} \boldsymbol{\nabla} \theta$ [14]. In Weyl semimetals $\boldsymbol{\nabla} \theta$ is the separation in momentum space between the Weyl nodes 
of right-handed and left-handed fermions. We observe that $\mu$ can be real or imaginary depending on the photon polarization, whereas in a nonchiral matter $\mu$ is always real. This is the origin of the distinct transition radiation pattern from chiral matter that we discuss in the next few paragraphs.

We start with case (i) representing spatially uniform matter. We assume that the boundary is located at $z=0$ and the particle moves in the $z$ direction, i.e., perpendicular to the boundary. At the boundary $\mu$ is discontinuous. In the ultrarelativistic limit, when $\mu$ can be treated as a small parameter, the photon wave function in the radiation gauge reads
$\boldsymbol{A}=\frac{1}{\sqrt{2 \omega V}} \boldsymbol{\epsilon}_{\lambda} e^{i \omega z+i \boldsymbol{k}_{\perp} \cdot \boldsymbol{x}_{\perp}-i \omega t} \exp \left(-i \frac{1}{2 \omega} \int_{0}^{z}\left(k_{\perp}^{2}+\mu^{2}\right) d z^{\prime}\right)$

where $\boldsymbol{\epsilon}_{\lambda}$ is the polarization vector such that $\boldsymbol{\epsilon}_{\lambda} \cdot \boldsymbol{k}=0$ and $V$ is the normalization volume. By the same token, the fermion wave function is

$\psi=\frac{1}{\sqrt{2 \varepsilon V}} u(p) e^{i \varepsilon z-i \varepsilon t} \exp \left\{i \boldsymbol{p}_{\perp} \cdot \boldsymbol{x}_{\perp}-i z \frac{\boldsymbol{p}_{\perp}^{2}+m^{2}}{2 \varepsilon}\right\}$,

where $\boldsymbol{p}$ and $\varepsilon$ are the fermion momentum and energy.

The scattering matrix element for the photon emission process is

$$
S=-i e Q \int \bar{\psi} \gamma^{\mu} \psi A_{\mu} d^{4} x=i(2 \pi)^{3} \delta\left(\omega+\varepsilon^{\prime}-\varepsilon\right) \delta\left(\boldsymbol{p}_{\perp}-\boldsymbol{k}_{\perp}-\boldsymbol{p}_{\perp}^{\prime}\right) \frac{\mathcal{M}}{\sqrt{8 \varepsilon \varepsilon^{\prime} \omega V^{3}}}
$$

where $Q$ is the fermion electric charge and the prime distinguishes the final fermion energy and momentum. The invariant amplitude reads

$$
\mathcal{M}=-e Q \bar{u}\left(p^{\prime}\right) \phi^{*} u(p) 2 \varepsilon x(1-x)\left\{\frac{-i}{q_{\perp}^{2}+\kappa_{\lambda}-i \gamma}-\frac{-i}{q_{\perp}^{2}+x^{2} m^{2}+i \gamma}\right\}
$$

where $x=\omega / \varepsilon$ is the fraction of the incident fermion energy carried away by the radiated photon, $\boldsymbol{q}_{\perp}=x \boldsymbol{p}-\boldsymbol{k}_{\perp}$; $\kappa_{\lambda}=x^{2} m^{2}+(1-x) \mu^{2}$ and $\gamma$ is the resonance width that depends on the system geometry, electrical conductivity, etc. The radiated photon spectrum can be computed as

$$
\frac{d N}{d^{2} q_{\perp} d x}=\frac{1}{(2 \pi)^{3}} \frac{1}{8 x(1-x) \varepsilon^{2}} \frac{1}{2} \sum_{\lambda, \sigma, \sigma^{\prime}}|\mathcal{M}|^{2},
$$

where $\sigma, \sigma^{\prime}$ are the fermion and antifermion spins. Substitution of Eq. (9) into Eq. (8) yields

$$
\frac{d N}{d^{2} q_{\perp} d x}=\frac{\alpha Q^{2}}{2 \pi^{2} x}\left\{\left(\frac{x^{2}}{2}-x+1\right) q_{\perp}^{2}+\frac{x^{4} m^{2}}{2}\right\} \sum_{\lambda}\left|\frac{1}{q_{\perp}^{2}+\kappa_{\lambda}-i \gamma}-\frac{1}{q_{\perp}^{2}+x^{2} m^{2}+i \gamma}\right|^{2} .
$$

For positive $\kappa_{\lambda}$, the photon spectrum (10) coincides with the standard formula for the transition radiation with $\mu$ being the plasma frequency [4]. However, the main contribution to the photon spectrum arises from the pole at $q_{\perp}^{2}=-\kappa_{\lambda}>0$, i.e., when $\kappa_{\lambda}$ is negative. Keeping only the term that is most singular at $\gamma \rightarrow 0$, we find the chiral transition radiation spectrum of photons [6]

$$
\frac{d N}{d^{2} q_{\perp} d x}=\frac{\alpha Q^{2}}{2 \pi^{2} x}\left\{\left(\frac{x^{2}}{2}-x+1\right) q_{\perp}^{2}+\frac{x^{4} m^{2}}{2}\right\} \frac{1}{\left(q_{\perp}^{2}+\kappa_{\lambda}\right)^{2}+\gamma^{2}} .
$$

It is remarkable that the spectrum is circularly polarized (In contrast, the ordinary transition radiation is linearly polarized [1].). Indeed, $\kappa_{\lambda}$ is negative only if $\lambda \sigma_{\chi}>0$ and $x<$ $\left[1+m^{2} /\left(\lambda \sigma_{\chi} \varepsilon\right)\right]^{-1}$. In other words, only one of the possible photon polarizations exhibits the resonant behavior, while the other one is suppressed. Whether the photon spectrum is right- or left-hand polarized depends on the sign of $\sigma_{\chi}$.

Since $\mu^{2} \approx-\lambda \sigma_{\chi} \omega$, the angular distribution of the photons peaks at the angle $\vartheta^{2}=q_{\perp}^{2} / \omega^{2}=-\kappa_{\lambda} / x^{2} \varepsilon^{2}$ with respect to the fermion momentum. If the fermion mass is negligible and bearing in mind that most photons are soft $(x \ll 1)$ we can estimate $\vartheta^{2} \approx \lambda \sigma_{\chi} / \omega$.

Applications. - 1. As the first application, consider jet emission from the quark-gluon plasma (QGP) with a homogenous chiral conductivity. QGP is isotropic at the scales of interest here; hence the corresponding case is (i). Jets in heavy-ion collisions are produced by the highly energetic color particles. If a jet is originated by a quark (as opposed to a gluon) we expect radiation of circularly polarized photons in a cone with the opening angle 
$\vartheta \sim \sqrt{\left|\sigma_{\chi}\right| / \omega}$ with respect to the jet momentum. The chiral conductivity is an unknown parameter. If we estimate it as $\sigma_{\chi} \sim 10 \mathrm{MeV}$, then $\omega=1 \mathrm{GeV}$ photons are emitted at the angle $\vartheta \sim 0.1$, provided that the jet energy $\varepsilon$ is much larger than $\omega$. Thus the observation of circularly polarized photons at angle $\vartheta$ to the jet direction would be an indication of the chiral transition radiation.

2. We have seen that the main feature of the transition radiation from chiral matter is the emergence of the resonance factor in Eq. (11). It arises entirely due to the energy and momentum conservation in a $1 \rightarrow 2$ process involving a photon with complex $\mu$. Thus we expect to see the same resonant factor as in Eq. (11) arising in the case (ii) which deals with an anisotropic matter. The calculation of the prefactor requires a more careful analysis that will be presented elsewhere. In the high energy limit Eq. (4) reduces to $\mu^{2} \approx-\lambda \omega b \cos \beta$, where $\beta$ is the angle between $\boldsymbol{b}$ and the photon momentum. The soft photon emission angle in the massless limit is $\vartheta^{2} \approx \lambda b \cos \beta / \omega$. Similarly to the previous case (i), the photon spectrum is circularly polarized. One can verify that now $\kappa_{\lambda}$ is negative only if $\lambda \cos \beta>0$ and $x<\left[1+m^{2} /(\lambda \varepsilon b \cos \beta)\right]^{-1}$. Thus the polarization direction depends on whether $\boldsymbol{b}$ points towards or away from the boundary. Furthermore, since $\mu^{2}$ is proportional to $\cos \beta$, the radiation is maximal when $\beta=0$ or $\pi$ and vanishes in the perpendicular direction. To estimate the characteristic radiation angle discussed above, consider a Weyl semimetal with $b=(\alpha / \pi) 80 \mathrm{eV}[17,18]$. An electron with energy about $\mathrm{GeV}$ moving parallel to $\boldsymbol{b}(\beta=0)$ would radiate, say, $\omega=$ $10 \mathrm{MeV}$ photons at $\vartheta=1.3 \times 10^{-4}$. This can be tested by injecting a beam of energetic electrons normal to a Weyl semimetal film and measuring the polarization and angular distribution of the photons emitted in a cone with the opening angle $\vartheta$ around the beam direction.

3. The chiral transition radiation emitted by protons traveling through the dark matter lumps [19] can be used to search for axionic dark matter. In this case $\dot{\theta}$ is proportional to the axion mass $m_{a}$ which is unknown but expected to be very small. The emission angle of the chiral transition radiation with respect to the direction of a cosmic ray is of the order of $\sqrt{c_{A} \theta_{0} m_{a} / \omega}$, where $\theta_{0}$ is the average value of $\theta$. Taking $\theta_{0} \sim 10^{-19}[20], m_{a} \sim 10^{-6} \mathrm{eV}$, and $\omega \sim 1 \mathrm{TeV}$ we obtain $\vartheta \sim 10^{-15}$. Measurement of photon spectrum emitted by a cosmic ray at such angles might be possible over the astronomical distances.

Summary.-In summary, we computed the transition radiation spectrum at the boundary between chiral matter and vacuum, given by Eq. (11) and argued that its unique features - the resonant enhancement at a characteristic angle $\vartheta$ and circular polarization - can be used as the direct measurement of the chiral anomaly in chiral matter or materials.

We thank Dima Kharzeev for valuable discussions. X. G. H. is supported by the Young 1000 Talents Program of China, NSFC through Grants No. 11535012 and No. 11675041 . K. T. is supported in part by the U.S. Department of Energy under Grant No. DE-FG0287ER40371.

[1] V. L. Ginzburg and I. M. Frank, Radiation of a uniformly moving electron due to its transition from one medium into another, Zh. Eksp. Teor. Fiz. 16, 15 (1946) [J. Phys. (USSR) 9, 353 (1945)].

[2] V.L. Ginzburg and V.N. Tsytovich, Several problems of the theory of transition radiation and transition scattering, Phys. Rep. 49, 1 (1979).

[3] V. N. Baier and V. M. Katkov, Quantum theory of transition radiation and transition pair creation, Phys. Lett. A 252, 263 (1999).

[4] D. Schildknecht and B. G. Zakharov, Transition radiation in quantum regime as a diffractive phenomenon, Phys. Lett. A 355, 289 (2006).

[5] S. Deser, R. Jackiw, and S. Templeton, Topologically massive gauge theories, Ann. Phys. (N.Y.) 140, 372 (1982); 281, 409 (2000); Erratum, Ann. Phys. (N.Y.) 185, 406(E) (1988).

[6] K. Tuchin, Radiative instability of quantum electrodynamics in chiral matter, Phys. Lett. B 786, 249 (2018).

[7] F. Wilczek, Two Applications of Axion Electrodynamics, Phys. Rev. Lett. 58, 1799 (1987).

[8] S. M. Carroll, G. B. Field, and R. Jackiw, Limits on a Lorentz and parity violating modification of electrodynamics, Phys. Rev. D 41, 1231 (1990).

[9] P. Sikivie, On the interaction of magnetic monopoles with axionic domain walls, Phys. Lett. 137B, 353 (1984).

[10] T. Kalaydzhyan, Chiral superfluidity of the quark-gluon plasma, Nucl. Phys. A913, 243 (2013).

[11] K. Tuchin, Electromagnetic field and the chiral magnetic effect in the quark-gluon plasma, Phys. Rev. C 91, 064902 (2015).

[12] K. Tuchin, Taming instability of magnetic field in chiral matter, Nucl. Phys. A969, 1 (2018).

[13] N. Yamamoto, Axion electrodynamics and nonrelativistic photons in nuclear and quark matter, Phys. Rev. D 93, 085036 (2016).

[14] Z. Qiu, G. Cao, and X. G. Huang, On electrodynamics of chiral matter, Phys. Rev. D 95, 036002 (2017).

[15] K. Fukushima, D. E. Kharzeev, and H. J. Warringa, The chiral magnetic effect, Phys. Rev. D 78, 074033 (2008).

[16] D. E. Kharzeev and H. J. Warringa, Chiral magnetic conductivity, Phys. Rev. D 80, 034028 (2009).

[17] S. Y. Xu et al., Discovery of a Weyl Fermion semimetal and topological Fermi arcs, Science 349, 613 (2015).

[18] B. Q. Lv et al., Experimental Discovery of Weyl Semimetal TaAs, Phys. Rev. X 5, 031013 (2015).

[19] C. Chang et al. (DES Collaboration), Wide-Field Lensing Mass Maps from Dark Energy Survey Science Verification Data, Phys. Rev. Lett. 115, 051301 (2015).

[20] P. W. Graham and S. Rajendran, New observables for direct detection of axion dark matter, Phys. Rev. D 88, 035023 (2013). 